\title{
Pour un monde durable : journée mondiale de la corrosion, 24 avril 2020
}

\author{
Philippe Marcus, \\ Président du Centre français de l'anticorrosion (CEFRACOR), ancien Président de l'European Federation of Corrosion (EFC), \\ Directeur du groupe de recherche de physico-chimie des surfaces de l'Institut de recherche de chimie Paris \\ (ENSCP Chimie ParisTech, CNRS, Université PSL), CEFRACOR, 28 rue Saint-Dominique, 75007 Paris, France
}

En France, la dégradation des matériaux sous l'effet de l'environnement a coûté, en 2019, plus de 80 milliards d'euros, et plus de $\mathbf{2 5 0 0}$ milliards de dollars dans le monde. Un effort accru de recherche scientifique et technologique et une meilleure prise en compte dans la formation, initiale et continue, sont indispensables. Il faut aussi sensibiliser le grand public et les pouvoirs publics. C'est l'objectif de la Journée mondiale de la corrosion, lancée par la World Corrosion Organisation, avec le soutien de la Fédération européenne de la corrosion et du Centre français de l'anticorrosion, programmée le 24 avril 2020.

Quatre-vingt-quatre milliards d'euros perdus : c'est le montant estimé du coût de la corrosion en France en 2019, en se basant sur le chiffre communément admis d'un coût de l'ordre de 3,5 \% du PIB.

Vieillissement, dégradation, rupture de matériaux métalliques utilisés dans des infrastructures routières, des pipelines transportant le pétrole, des conduites de gaz, des moyens de transport (automobiles, trains, avions), tout cela résulte principalement de l'interaction des surfaces des matériaux avec leur environnement, du fait de la réaction chimique de corrosion. Outre ce coût exorbitant, des problèmes majeurs sont liés à la dégradation des matériaux sous l'effet de l'environnement : atteinte à la fiabilité des installations, menace pour la sécurité des biens et des personnes, problèmes de santé.

Il faut prévenir ou tout au moins ralentir la corrosion : une prise de conscience est nécessaire, et celle-ci ne doit pas se limiter aux spécialistes des matériaux, déjà largement avertis, mais doit être beaucoup plus générale, et toucher les décideurs, les pouvoirs publics et la population (des plus jeunes aux plus âgés !).

Une méconnaissance des aspects scientifiques et techniques des phénomènes de corrosion, certes complexes, et une sous-estimation de l'importance des risques et des coûts sont trop souvent constatées.

Quels sont les leviers d'action ? La réponse est : éducation, recherche, innovation.

Éducation au sens large, qui doit inclure formation initiale à tous les niveaux, du primaire aux universités et aux écoles d'ingénieurs, et formation continue (des techniciens et des ingénieurs).

Recherche académique et industrielle : une recherche de haut niveau est déjà en place dans les laboratoires publics et les grands organismes (universités, écoles d'ingénieurs, CNRS, CEA).

La plupart des grands groupes français ont une activité de R\&D sur la protection contre la corrosion. Cependant, ces efforts doivent être accrus (tout comme l'effort global de recherche en France !).

Les recherches fondamentales sur les mécanismes de corrosion doivent prendre en compte toutes les échelles, échelle d'espace, de l'échelle atomique ou nanométrique (à laquelle les phénomènes de corrosion démarrent sur la surface d'un matériau) à l'échelle macroscopique(à laquelle arrivent les dégradations, alors souvent irréversibles), et échelle de temps, fonction de la durée de vie visée (de quelques secondes pour le lancement d'une fusée, à quelques centaines de milliers d'année pour les matériaux de stockage des déchets nucléaires). Les connaissances nouvelles ainsi produites doivent servir de base aux recherches plus appliquées et au développement de nouvelles technologies.

Au-delà des domaines classiques d'utilisation des matériaux métalliques qui concernent un large spectre de secteurs industriels, une meilleure protection des matériaux contre la corrosion est un facteur clé dans le développement de nouvelles applications, notamment pour assurer la transition énergétique (piles à combustible, batteries, photovoltaïque). Ainsi, à titre d'exemple, dans la perspective d'une politique de l'énergie mettant en jeu l'hydrogène, de nombreux problèmes de matériaux apparaissent et la protection contre la corrosion constitue un enjeu majeur.

Des actions de sensibilisation auprès du grand public et des pouvoirs publics sont également indispensables.

Pour favoriser la prise de conscience des enjeux sociétaux de la corrosion et mobiliser les principaux acteurs, la World Corrosion Organization (WCO) a lancé en 2010 une Journée mondiale de la corrosion. En 2020, cette journée mondiale a été fixée au 24 avril. 